
\title{
R Resercrch Suare \\ Effects of Early Mask Mandates and Other Policy Interventions on COVID-19 Infections
}

\section{Brian An}

Georgia Tech

Simon Porcher ( $\nabla$ porcher.iae@univ-paris1.fr)

Université https://orcid.org/0000-0001-6614-0338

Shui Yan Tang

Sol Price School of Public Policy, USC

Emily Kim

Georgia Tech

\section{Article}

Keywords: mask mandates, COVID-19, government

Posted Date: January 5th, 2021

DOI: https://doi.org/10.21203/rs.3.rs-139339/v1

License: @ (i) This work is licensed under a Creative Commons Attribution 4.0 International License. Read Full License 


\section{Abstract}

How do different government mandates compare in their short and long-term efficacies in curbing COVID19 infections? Does a mandate's early adoption enhance its eventual efficacy? Using worldwide data on the daily evolution of government mandate adoptions and COVID-19 infections from January to July 2020 , we found that, compared with five other commonly adopted mandates, early adoption of the mask mandate has the strongest short and long-term effect on curbing infections. Mass gathering bans and school closures have some effect but take more time to manifest their efficacy. Domestic lockdowns and restaurant closures have the least effect. When designing measures for curbing COVID-19 infections, governments must weigh the timing and relative efficacies of alternative policy instruments.

\section{Introduction}

Before the widespread availability of an effective vaccine, the only viable approach to slow the spread of SARS-CoV-2 (COVID-19) is to use government-imposed non-pharmaceutical interventions (NPIs) such as social distancing, mask mandates, contact tracing, and closures of schools and businesses. Individual studies have assessed the efficacies of different NPIs. ${ }^{1-9}$ Yet, despite their demonstrated effectiveness, many NPIs hurt the economy and other aspects of social and personal wellbeing. ${ }^{7}$ Still, little is known about how NPIs compare in their relative efficacies. ${ }^{6,8,10}$ Suppose mandatory mask-wearing is more effective than other NPIs. If it is imposed shortly after the initial outbreak, it may be unnecessary to mandate more drastic measures such as domestic lockdowns and business closures. ${ }^{8}$ It is, however, difficult to compare the efficacies of NPIs without conducting a randomized controlled trial. ${ }^{10}$ Before such experiments are available, is it feasible to use existing data on worldwide NPI adoptions to gauge their relative efficacies?

A related policy question is whether the early adoption of an NPI enhances its eventual efficacy. Transmission rates among asymptomatic or pre-symptomatic groups may not be significantly different from those among symptomatic patients. ${ }^{11,12}$ Yet COVID-19 has shown differential transmission rates by age groups. ${ }^{13}$ For example, epidemic data and simulation models indicate lower transmissibility among children than adults. If so, closing down schools early on alone may have less effect on total infections ${ }^{3}$ than other NPIs targeting older adults. ${ }^{13}$ Such epidemiological factors may affect whether early adoption is crucial for an NPI to work. Our research addresses this critical policy question by asking which NPI benefits the most from early adoption.

We leverage unique worldwide data that record the daily evolution of NPI adoption and COVID-19 infections between January 1 and July 15, 2020, a timeframe generally considered the first wave of the global pandemic. ${ }^{14}$ Specifically, we identify the timing of NPI adoption and compare its early intervention effect to that of late and non-adoption. Our analysis focuses on the associations between COVID-19 infections (both daily new cases and total cases per million) and six NPIs commonly adopted across the globe: (1) mask mandates, (2) international travel restrictions, (3) domestic lockdowns, (4) mass 
gathering bans, (5) restaurant closures, and (6) school closures. The data also allow us to distinguish the differential effects of nationwide (i.e., strict) mandates from regional (i.e., partial) ones.

Figure 1 presents the worldwide data on the number of days taken for each NPI before its adoption as a strict mandate. The bar represents the median number of days taken. As indicated by interquartile range boxes, there is substantial heterogeneity across nations for each strict mandate. Yet taking the median as the baseline, countries generally mandated school closures first and mask mandates last. Also, the mask mandate was the least frequently utilized among the six NPIs during the first wave of the pandemic. When considering the six mandates together, the global median for NPI adoption time is 14 days. We thus define early NPI adoption for all six mandates as being taken within 14 days after the first infection.

\section{Results}

\section{Short-term effects of NPI adoption}

Figure 2 shows the short-term effect of strict NPI mandates on new infection rates 5, 9, 12, and 21 days after their adoption, using fixed-effects models (Methods). Three major findings are worth noting. First, only two NPIs show consistently significant durable effects over all time lags between 5 and 21 days: mask mandates and mass gathering bans. Specifically, mask mandates show the greatest effect until 12 days. Second, both domestic lockdowns and restaurant closures do not seem to help contain virus transmission in the short-term, showing no significant effect in any of the five different time lags. Lastly, while the effect of mask mandates appears immediately and then decreases monotonically over time, mass gathering bans and school closures show otherwise; their effects need more time to materialize. The full results, including 30 days lag, are reported in Table S1 in Supplementary Materials.

Table S2 in Supplementary Materials reports the results of a similar model with dummies for the different scales of NPI implementation (strict, partial, or not implemented). Unlike Fig. 2, in which the implementation scale takes three different values, the model uses dummies instead to differentiate the effects of partial versus strict measures (Methods). Again, strict mask mandates show the highest efficacy in containing the virus until 12 days.

164 countries in all models. Fixed-effects regression for panel data used for estimation. Robust standard errors clustered by subcontinents. Within R-squared is between 0.863 and 0.867 .

95\% (bold) and 99\% (thin) confidence intervals are reported around the coefficient.

\section{Long-term effects of early NPI adoption: Cross-sectional data analysis}

To measure early NPI adoption's long-term effects in the cross-sectional country-level data, we chose total infections per million between the 90th and 120th day. This timeframe allows for measuring long-term effects during the first wave while covering most countries worldwide. Figure 3 shows that countries 
mandating mask-wearing within two weeks of the first COVID-19 infection had fewer total infections in later days (i.e., between the 90th and 120th day) than those that did not. The effect size is substantial as it translates to 0.61 standard deviations of fewer infections in the 134 country samples. With each NPI mandate coded as 0 (no adoption), 0.5 (partial/regional), and 1 (strict/national), the model suggests that countries with mask mandates within the first two weeks saw 1.22 standard deviations fewer infections per million than those with no such mask mandates. By contrast, the other five NPIs are not significant predictors of population-adjusted infections in the later days during the first wave. Most controls show expected signs, but none is statistically distinguishable from zero, except for GDP per capita.

Table S3 in Supplementary Materials shows that the negative correlation between early mask mandates and total infections per million remains robust regardless of other known factors on infections and mortality rates, such as government effectiveness, number of hospital beds, and other demographic health risk indicators. It also remains robust after considering whether a country ever mandated the six NPIs during the analysis period; this further purges the effect of early NPI adoptions from that of their mere presence. Additional robust analyses reported in Table S4 and S5 further assure that the key correlations are unaffected by potential outliers or an alternative timeframe for assessing long-term effects.

\section{Long-term effects of early NPI adoption: Panel data analysis}

To address concerns associated with unobservable confounding factors in the preceding analysis, we use panel data by comparing the long-term effects of early mask mandates with those of other NPIs among three groups of countries: 1 ) those implementing a strict mandate early on (i.e., early adopters), 2) those implementing a strict mandate after the first two weeks (i.e., late adopters), and 3) those implementing no strict mandate at all during the study period (i.e., non-adopters as a comparison group). Capturing a shock at only one point in time, namely the date the mandates came into effect nationally, the analysis estimates the long-term consequences of NPIs relative to their absence, zeroing particularly on the potential importance of early actions.

Figure 4 presents the results from fixed-effects (upper panel) and random-effects (lower panel) models (also see Table S6). Consistent with the preceding analysis, these panel data results indicate that countries with an early nationwide mask mandate saw significantly fewer total infections over time than those without such a mandate during the first wave, on average, by as many as 1442 or 1668 per million population $(p<0.05)$. These effect sizes are also substantial as they translate to 0.61 and 0.71 standard

deviations of fewer total infections in the 164 country samples. Taken together, the estimations from both fixed-effects and random-effects models provide supporting evidence of the persistent effect of early mask mandates in controlling virus transmission over time.

\section{Discussion}

Policymakers worldwide are increasingly seeking evidence-based policy instruments to combat the COVID-19 pandemic. While anecdotal evidence and laboratory experiments have shown the efficacy of 
mask-wearing in reducing infection chances, $4,5,15,16$ the strength of the mandate's short and long-term effects relative to other NPIs has not been examined in observational studies.

Our research fills this gap by leveraging a unique worldwide dataset to show that not all NPIs are equally effective during the first wave of the pandemic. ${ }^{8}$ Among the six most commonly adopted NPIs worldwide, mask mandates are the most effective in immediately curbing new infections. By contrast, domestic lockdowns and restaurant closures show no immediate effect, suggesting that they are, relatively speaking, the least effective NPIs in containing the virus in the short-term. Also, mass gathering bans and school closures take more time to manifest their efficacy. Hence policymakers need to be aware of the differential effects of various NPIs and their time to achieve public health goals. Each instrument's benefits and costs should be gauged against the expected effects and required timeframe.

Moreover, it is critically important to adopt some NPIs early on. ${ }^{9}$ Specifically, this research shows that countries with strict mask mandates adopted within the first two weeks saw far fewer infections subsequently than those that acted later or took no actions at all. These results highlight the role of early preparedness and agile actions as critical components of successful pandemic management. $5,6,9,17,18$ Notably, mask mandate - which are less costly for the economy than domestic lockdowns or restaurant closures - is the most effective in the long term among other instruments if adopted at the beginning of the outbreak. This article provides a valuable lesson for countries to prepare for the next pandemic. Without early adoption of the most effective NPI instruments such as mask mandates, many governments may keep repeating the same mistakes in pandemic management.

Our analysis provides valuable insights because of the varying transmission dynamics and government responses in different countries. The research framework is similar to a quasi-natural experiment in which different countries might implement different sets of NPIs at different times. The results are robust when considering alternative modelling specifications, alternative ways of coding the variables of interest (see the Supplementary Tables), and short-term vs. long-term perspectives. The study's core result is that mask mandates, which involve fewer economic and social costs, are a more effective intervention than other more costly NPIs to contain the spread of COVID-19. Our results are in line with the results of other studies finding a strong effect of mask mandates on COVID-19 containment ${ }^{19,20}$.

Our results do not mean that governments should abandon the implementation of some NPIs. The estimated relationships are additive effects controlling for other NPIs; they should be read as relative effects, not absolute ones. Hence, it would be misleading to interpret some non-significant NPIs as not effective at all. For example, domestic lockdowns, as shown in other recent studies ${ }^{8}$, have a limited effect on COVID-19 containment. Meanwhile, early evidence on a smaller number of countries suggests that they are the most effective $\mathrm{NPI}^{21}$. Our results indicate that the additive effect of domestic lockdowns, which are the ultimate ban, can be limited, particularly when all other NPIs are already in place.

It is challenging to assess the relative efficacies of various NPIs, which are often implemented simultaneously in different contexts. Hence, the coefficients should be interpreted carefully. Second, this 
study is not estimating the optimal time for early NPI adoption. Instead, the 14-day early adoption window was chosen as a result of data distribution analysis. The virus, however, reached various countries at different times. Hence the definition of early adoption may have to be adjusted based on each country's unique circumstances. Third, while our study focuses on early adoption, many countries repeatedly relaxed strict and partial NPIs and re-imposed them upon virus surges. Future research may further examine how such dynamic choices have affected subsequent infection trajectories. Finally, while our data collection allows us to control for each instrument's implementation scale, it does not allow us to control for whether the population respects the NPIs and how governments enforce them.

\section{Methods}

Data

COVID-19 cases. Data on cases and deaths come from the Johns Hopkins Coronavirus Resource Center (https://coronavirus.jhu.edu/). The data are published daily since January $1 \mathrm{st}, 2020$. The data allow us to compute the cumulative deaths and cases on a daily basis. To compare across countries of different population sizes, we also count cases per million inhabitants or total cases per million inhabitants to build our main dependent variables.

In the analysis of short-term NPI effects on COVID-19, we account for the real trend of new cases and fewer cases reported during weekends by measuring the mean of cases per million inhabitants reported in the last seven days. For a given day $t$ and a country $c$, the number of new cases per million inhabitants is thus computed as

\section{$\operatorname{NewCases}_{c, t}=\sum_{l=0}^{6} \operatorname{Cases}_{c, t-l}$.}

with / the number of lags and Cases the reported cases per million inhabitants. We apply the same smoothing procedure for total cases. We use these smoothed variables to compute the new case rate as RateNewCases $_{d, c}=\frac{\text { NewCases }_{d, c}}{\text { TotalCases }_{d, c}} \times 100$.

The new case rate is a good proxy for the short-term pandemic scale and is used as a dependent variable. In the long-term analysis, we assess NPI effects by focusing on total cases per million inhabitants, which are good proxies for government capacity to contain the pandemic during a period. In the model in which we use country-level cross-sectional data, we use the average total cases per million between the 90th and 120th days after the first reported case as the dependent variable; this is to control for the differences in pandemic trends across countries. Our assessment thus compares countries within the same time interval.

Non-pharmaceutical interventions and the timing of their implementation. Data on NPIs are obtained from the Response2covid19 dataset. ${ }^{22}$ We particularly consider six NPIs that have strong potential 
effects on virus containment. The six NPIs considered are mask mandates, international travel restrictions, domestic lockdowns, mass gathering bans, restaurant closures, and school closures. Mask mandates are obligations to wear masks in public spaces. International travel restrictions are bans on international flights or border closures, except for cargo flights or repatriation. Domestic lockdowns are stay-at-home orders. Mass gathering bans refer to limitations on public or private gatherings. Restaurant and school closures refer to these facilities' closures.

The NPIs are coded in a three-scale format. NPIs can be mandated for the entire population ("strict" intervention), for only a subpopulation ("partial" intervention), e.g., in a localized area or for a given category of the population, or not implemented at all. This differentiation is essential because strict NPIs will be useless in some cases, for example, if the virus is contained in a given region. The categorization allows us to compare different clusters of countries-being strict vs. being partial in implementing NPIs vs. not implementing. NPIs are coded 0 if not implemented, 0.5 if partially implemented, and 1 if strictly implemented.

We consider different timeframes to analyze short-term NPI effects. Because of the incubation period, NPIs often do not have immediate effects. To consider their short-term effects, we use different lags - 5 , $9,12,21$, and 30 days. The first lags - between 5 and 12 days - are selected because most patients experience symptoms between the 5th and 12th days. ${ }^{23}$ The other lags, 21 and 30 days, are used to see if the NPIs have more lasting effects.

In the long-term analysis, we focus on NPI timing, an essential factor in virus containment. ${ }^{1,9}$ To operationalize this, we include dummies capturing whether a country has implemented a given NPI early on. We define early implementation as having the NPI in place within 14 days after the first reported case, as this is the global median for all six NPIs (see Fig. 1 in the main document).

Control variables. In the short-term analysis, we use various controls capturing the pandemic's dynamics. Some controls are standard in SIR (Susceptible, Infected and Recovered) epidemiological models. For example, we account for the smoothed cumulative total cases and deaths reported at date $t$ in a given country $c$. We also add two extra controls to improve model estimation as they indicate where a country stands at a particular time during the pandemic: We first include the lagged value of the dependent variable, which is the new case rate. We also include the logged number of days since January $1 \mathrm{st}, 2020$, a time trend accounting for the timing of the virus's worldwide spread, and the importance of knowledge and experience in containing the virus.

In the long-term analysis with country-level cross-sectional data, different socio-economic variables from the World Bank are included. Following recent studies, ${ }^{24-27}$ we include several variables capturing national healthcare capacities -hospital beds per 1,000 inhabitants and health expenditures as a percentage of GDP in 2018. We also use controls capturing the population's health risk-the percentage of the population with diabetes and the percentage being overweighed. We also add the median age of the population to indicate its sensitivity to COVID-19. Note that this variable is correlated strongly with $\%$ of 
older population aged 65 or more, with $r=0.913$ in our main estimation reported in Fig. 3 . We thus do not include percent of older population aged 65 or more. We finally include controls capturing a country's developmental level-GDP per capita and government effectiveness.

\section{Regression techniques}

The short-term NPI effect on COVID-19 infections is assessed using fixed-effects regressions. As we use longitudinal panel data, the within estimator allows us to model each country's mean as a group-specific fixed quantity. This specification has two advantages. First, the fixed-effects model controls for all the time-invariant country-specific features such as experience in handling pandemics or the overall quality of government and health systems. Second, the within estimator relates changes in the dependent variable to effects from time-variant characteristics such as NPI adoptions.

The long-term NPI effect on COVID-19 infections is first assessed using country-level cross-sectional data with ordinary least squared regressions. To account for unobservable, beyond-country factors that are associated with both the independent and dependent variables, the model includes continent fixed-effects (Africa, America, Asia, Europe and Pacific). While this method is useful for comparing cross-country variations, it still does not control for other unobservable confounding factors specific to each country. For instance, countries adopting mask mandates early on may have characteristics different from others, such as culture, prior pandemic experience, domestic mask production capacity, and public information campaign capacity, among others, which are not captured by the five continent-fixed effects. If so, the estimated relationship could be due to such unobservable factors rather than early mask mandates. Besides, since a cross-sectional analysis only focuses on differences between countries, it does not tell how COVID-19 infections would be different if a country adopted the mask mandate earlier than other NPIs. Hence we assess long-term NPI effects on infections by using panel data with longitudinal least squared regressions methods. Here, the models include country fixed-effects or random-effects.

In this panel data structure using within-country variations (i.e., country fixed-effects models), each NPI adoption captures a shock at only one point in time, namely, the date the mandate came into effect nationally. Yet the dependent variable "total cumulative infections per million" evolves daily for all data points. Given that the two sets of variables were measured at different times, and the country-level factors had remained constant in the fixed-effects model, it is challenging to estimate the long-term effect of early mandate adoption. In other words, the model estimates the pure effect of NPI adoption timing using the variations within countries while taking into account differences in their inherent capacities for implementing such an instrument.

All models are estimated with robust standard errors clustered at the subcontinent level, which is at a greater geographical scale than a country, to avoid potential downward biases. ${ }^{28}$ The rationale is that the error terms may not be perfectly independent of other neighboring countries in the same subcontinent given that there may be more significant virus transmission and policy diffusion among proximate countries. ${ }^{29,30}$ We thus use 19 subcontinents: (1) North America, (2) the Caribbean and Central America, 
(3) South America, (4) East Asia, (5) Southeast Asia, (6) South Asia, (7) Central Asia, (8) West Asia, (9) Oceania, (10) Northern Europe, (11) Eastern Europe, (12) Southern Europe, (13) Western Europe, (14) Eastern Africa, (15) Southern Africa, (16) Northern Africa, (17) Central Africa, (18) Western Africa, and (19) the Middle East. This subcontinent-level clustering generates more conservative standard errors than country-level clustering while still allowing for considerable degrees of freedom.

\section{References}

1. Ferguson, N., Laydon, D., Nedjati-Gilani, G., Imai, N., Ainslie, K., Baguelin, M., ... \& Dighe, A. (2020). Report 9: Impact of non-pharmaceutical interventions (NPIs) to reduce COVID19 mortality and healthcare demand. Imperial College London, 10, 77482. https://doi.org/10.25561/77482.

2. Cheng, H. Y., Jian, S. W., Liu, D. P., Ng, T. C., Huang, W. T., \& Lin, H. H. (2020). Contact tracing assessment of COVID-19 transmission dynamics in Taiwan and risk at different exposure periods before and after symptom onset. JAMA Internal Medicine.

https://doi.org/10.1001/jamainternmed.2020.2020.

3. Viner, R., Russell, S., Croker, H., Packer, J., Ward, J., Stansfield, C., ... Booy, R. (2020). School Closure and Management Practices during Coronavirus Outbreaks including COVID-19: A Rapid Narrative Systematic Review. Lancet. https://doi.org/10.1016/S2352-4642(20)30095-X.

4. Betsch, C., Korn, L., Sprengholz, P., Felgendreff, L., Eitze, S., Schmid, P., \& Böhm, R. (2020). Social and behavioral consequences of mask policies during the COVID-19 pandemic. Proceedings of the National Academy of Sciences, 117:21851-21853. https://doi.org/10.1073/pnas.2011674117.

5. ${ }^{5}$ Xu, J., Hussain, S., Lu, G., Zheng, K., Wei, S., Bao, W., \& Zhang, L. (2020). Associations of Stay-atHome Order and Face-Masking Recommendation with Trends in Daily New Cases and Deaths of Laboratory-Confirmed COVID-19 in the United States. Exploratory Research and Hypothesis in Medicine, 1-10. https://doi.org/10.14218/ERHM.2020.00045.

6. Anderson, R. M., Heesterbeek, H., Klinkenberg, D., \& Hollingsworth, T. D. (2020). How will countrybased mitigation measures influence the course of the COVID-19 epidemic? Lancet, 395:931-934. https://doi.org/10.1016/S0140-6736(20)30567-5.

7. Spelta, A., Flori, A., Pierri, F., Bonaccorsi, G., \& Pammolli, F. (2020). After the lockdown: simulating mobility, public health and economic recovery scenarios. Nature Scientific Reports, 10(1): 1-13. https://doi.org/10.1038/s41598-020-73949-6

8. Haug, N., Geyrhofer, L., Londei, A., Dervic, E., Desvars-Larrive, A., Loreto, V., ... \& Klimek, P. (2020). Ranking the effectiveness of worldwide COVID-19 government interventions. Nature Human Behaviour, 1-10. https://doi.org/10.1038/s41562-020-01009-0.

9. Pei, S., Kandula, S., \& Shaman, J. (2020). Differential Effects of Intervention Timing on COVID-19 Spread in the United States. Science Advances, 6(49): eabd6370. https://doi.org/10.1126/sciadv.abd6370.

10. Haushofer, J., \& Metcalf, C. J. E. (2020). Which interventions work best in a pandemic? Science, 368:1063-1065. https://doi.org/10.1126/science.abb6144. 
11. Lee, S., Kim, T., Lee, E., Lee, C., Kim, H., Rhee, H., ... Choo, E. J. (2020). Clinical course and molecular viral shedding among asymptomatic and symptomatic patients with SARS-CoV-2 infection in a community treatment center in the Republic of Korea. JAMA Internal Medicine, 180: 1447-1452. https://doi:10.1001/jamainternmed.2020.3862.

12. Savvides, C., \& Siegel, R. (2020). Asymptomatic and presymptomatic transmission of SARS-CoV-2: A systematic review. MedRxiv. https://dx.doi.org/10.1101\%2F2020.06.11.20129072.

13. Davies, N. G., Klepac, P., Liu, Y., Prem, K., Jit, M., Eggo, R. M., \& CMMID COVID-19 working group. (2020). Age-dependent effects in the transmission and control of COVID-19 epidemics. MedRxiv. https://doi.org/10.1038/s41591-020-0962-9.

14. Cacciapaglia, G., Cot, C. and Sannino, F. (2020). Second wave COVID-19 pandemics in Europe: a temporal playbook. Nature Scientific Reports, 10(1):1-8. https://doi.org/10.1038/s41598-020-726115.

15. Peeples, L. (2020). What the data say about wearing face masks. Nature, 586:186-189. https://doi.org/10.1038/d41586-020-02801-8.

16. Leung, N. H., Chu, D. K., Shiu, E. Y., Chan, K. H., McDevitt, J. J., Hau, B. J., ... Seto, W. H. (2020). Respiratory virus shedding in exhaled breath and efficacy of face masks. Nature Medicine, 26:676680. https://doi.org/10.1038/s41591-020-0843-2.

17. https://doi.org/10.1073/pnas.2011674117.

18. Moon, M. J. (2020). Fighting Against COVID-19 with Agility, Transparency, and Participation: Wicked Policy Problems and New Governance Challenges. Public Administration Review. https://doi.org/10.1111/puar.13214.

19. Perry, R. W., Lindell, M. K., \& Tierney, K. J. (Eds.). (2001). Facing the unexpected: Disaster preparedness and response in the United States. Joseph Henry Press.

20. Wey, L. and Wehby, G. L. (2020). Community use of face mask and COVID-19: Evidence from a natural experiment of State mandates in the US. Health Affairs, 39(8):1419-1425.

21. Zhang, R., Li, Y., Zhang, A.L., Wang, Y. and Molina, M.J. (2020). Identifying airborne transmission as the dominant route for the spread of COVID-19. PNAS, 117(26):14857-14863.

22. Flaxman, S., Mishra, S. [... Bhatt, S. (2020). Estimating the effects of non-pharmaceutical interventions on COVID-19 in Europe. Nature, 584: 257-261.

23. Porcher, S. (2020). Response2covid19, a dataset of governments' responses to COVID-19 all around the world. Scientific Data 7:423. https://doi.org/10.1038/s41597-020-00757-y.

24. Lauer, S. A., Grantz, K. H., Bi, Q., Jones, F. K., Zheng, Q., Meredith, H. R., ... Lessler, J. (2020). The incubation period of coronavirus disease 2019 (COVID-19) from publicly reported confirmed cases: estimation and application. Annals of Internal Medicine 172(9): 577-582. https://doi.org/10.7326/M20-0504.

25. Liang, L.L., Tseng, C.H., Ho, H.J. and Wu, C.Y.. (2020). COVID-19 mortality is negatively associated with test number and government effectiveness. Nature Scientific Reports, 10(1):1-7. https://doi.org/10.1038/s41598-020-68862-x. 
26. Holman, N., Knighton, P., Kar, P., O'keefe, J., Curley, M., Weaver, A. et al. (2020). Risk factors for COVID19-related mortality in people with type 1 and type 2 diabetes in England: a population-based cohort study. Lancet. https://doi.org/10.1016/S2213-8587(20)30271-0.

27. Qian, Lei., Hong, V., Wei, R. et al. (2020). Obesity and Mortality Among Patients Diagnosed With COVID-19: Results From an Integrated Health Care Organization. Annals of Internal Medicine, 173:773-781. https://doi.org/10.7326/M20-3742.

28. Khan, J. R., Awan, N., Islam, M. M., \& Muurlink, O. (2020). Healthcare Capacity, Health Expenditure, and Civil Society as Predictors of COVID-19 Case Fatalities: A Global Analysis. Frontiers in Public Health, 8:347. https://doi.org/10.3389/fpubh.2020.00347.

29. Cameron, A. C., \& Miller, D. L. (2015). A practitioner's guide to cluster-robust inference. Journal of Human Resources, 50(2): 317-372. https://doi.org/10.3368/jhr.50.2.317.

30. Sebhatu, A., Wennberg, K., Arora-Jonsson, S., \& Lindberg, S. I. (2020). Explaining the homogeneous diffusion of COVID-19 nonpharmaceutical interventions across heterogeneous countries.

Proceedings of the National Academy of Sciences 117(35): 21201-21208.

https://doi.org/10.1073/pnas.2010625117.

31. Mistur, E., Givens, J. W., \& Matisoff, D. (2020). Policy contagion during a pandemic. Available at SSRN 3662444. https://papers.ssrn.com/sol3/papers.cfm?abstract_id=3662444.

\section{Figures}

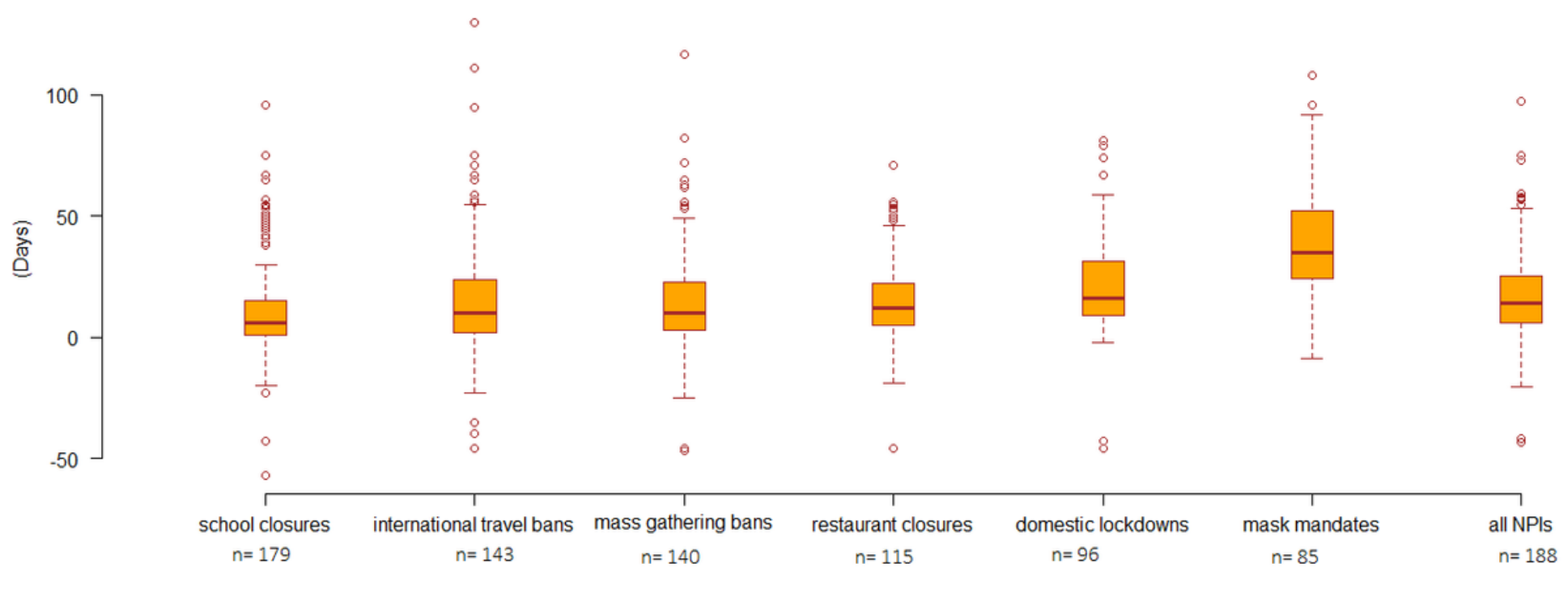

Figure 1

Number of days taken for strict NPI adoption worldwide Number of days taken for strict NPI adoption worldwide 

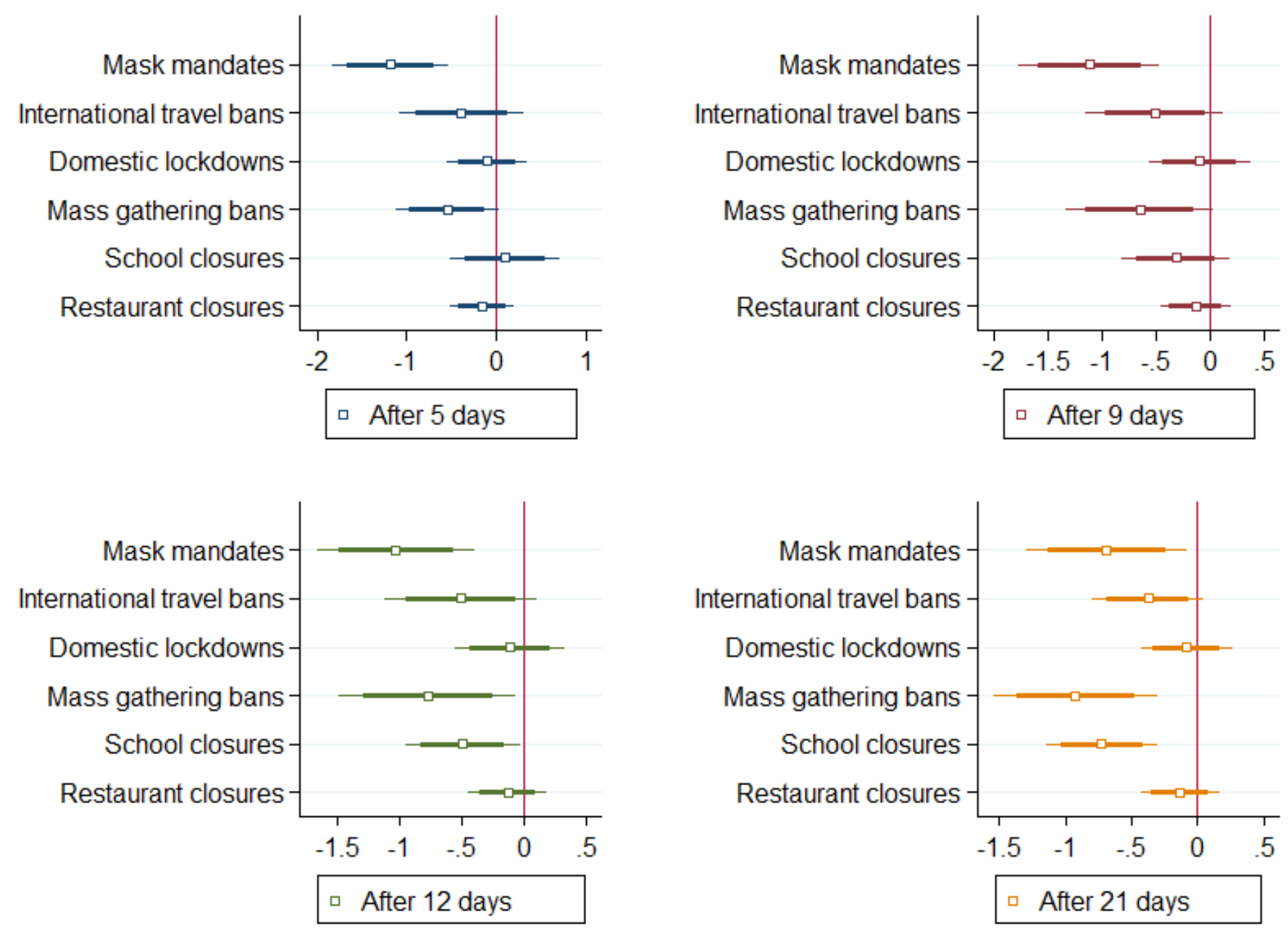

\section{Figure 2}

Short-term effects of NPI adoption on new case rates Notes: This table summarizes the impact of NPIs on the rate of new cases. Full results are reported in Table S1 in Supplemental Materials.

$n=21,126 \sim 21,155$ country-day pairs, covering 164 countries in all models. Fixed-effects regression for panel data used for estimation. Robust standard errors clustered by subcontinents. Within R-squared is between 0.863 and 0.867 . 95\% (bold) and 99\% (thin) confidence intervals are reported around the coefficient. 


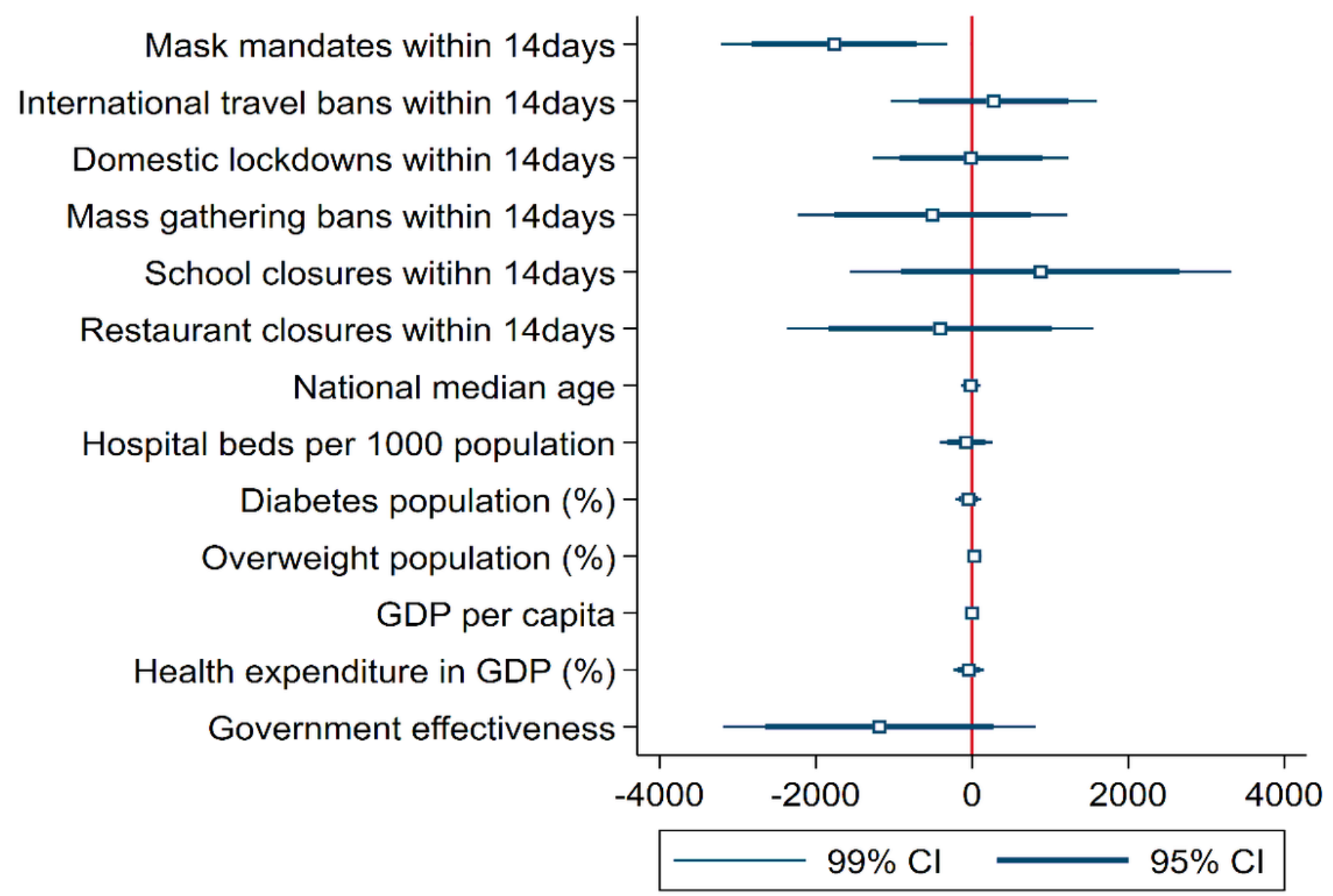

Figure 3

Long-term effects of early NPI adoption (cross-sectional data analysis) Notes: Estimates represent the effects between the 90th and 120th days. $n=134$ countries. Ordinary least squares regression used for estimation. Subcontinent fixed-effects regression for cross-sectional data used for estimation. Robust standard errors clustered by subcontinents. R-squared is 0.519 and adjusted R-squared is 0.448 . 

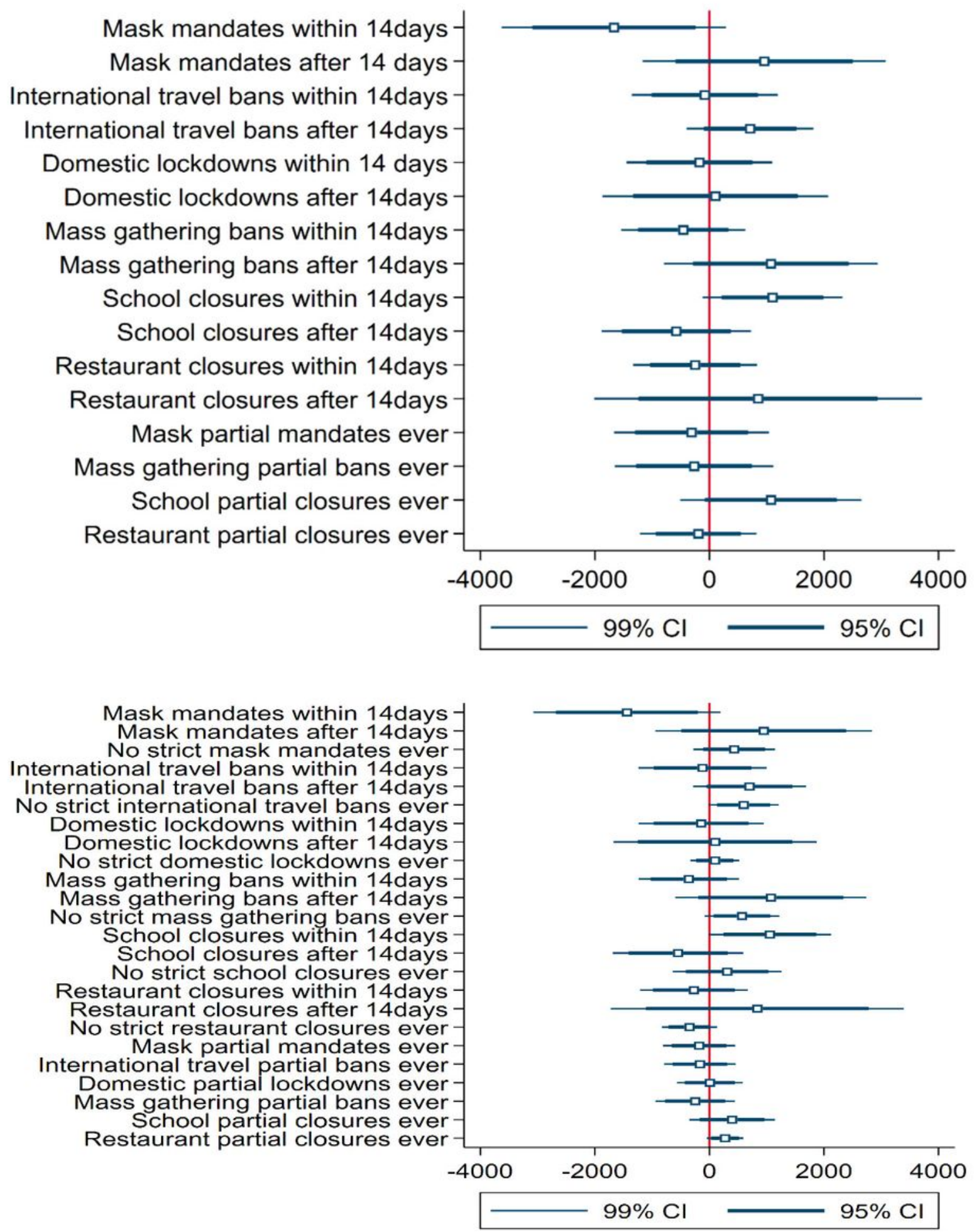

Figure 4

Long-term effects of early NPI adoption (panel data analysis) (Top) Panel A. Country Fixed Effects Model (Bottom) Panel B. Country Random Effects Model Notes: n=24,684 country-day pairs, covering 164 countries in both panels. Ordinary least squares regression for panel data used for estimation. Robust standard errors clustered by subcontinents. Within R-squared is 0.185 for fixed-effects model and overall R-squared is 0.151 for random-effects model. The results are also reported in Table S6. 


\section{Supplementary Files}

This is a list of supplementary files associated with this preprint. Click to download.

- SupplementaryMaterialsNHB.docx 